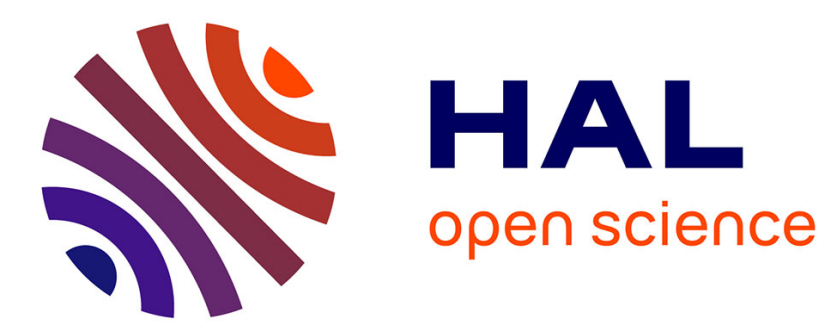

\title{
Electronic transport in strongly coupled and fully ionized $\mathrm{H}$-He mixtures
}

D. Leger, C. Deutsch, J.P. Hansen

\section{To cite this version:}

D. Leger, C. Deutsch, J.P. Hansen. Electronic transport in strongly coupled and fully ionized H-He mixtures. Journal de Physique Lettres, 1981, 42 (21), pp.459-462. 10.1051/jphyslet:019810042021045900 . jpa-00231976

\section{HAL Id: jpa-00231976 https://hal.science/jpa-00231976}

Submitted on 1 Jan 1981

HAL is a multi-disciplinary open access archive for the deposit and dissemination of scientific research documents, whether they are published or not. The documents may come from teaching and research institutions in France or abroad, or from public or private research centers.
L'archive ouverte pluridisciplinaire HAL, est destinée au dépôt et à la diffusion de documents scientifiques de niveau recherche, publiés ou non, émanant des établissements d'enseignement et de recherche français ou étrangers, des laboratoires publics ou privés. 


\title{
Electronic transport in strongly coupled and fully ionized H-He mixtures
}

\author{
D. Leger, C. Deutsch \\ Laboratoire de Physique des Plasmas $\left({ }^{*}\right)$, Université Paris-XI, Bâtiment 212, 91405 Orsay, France
}

and J. P. Hansen

Laboratoire de Physique Théorique des Liquides (*), Université de Paris-VI, 75230 Paris Cedex 05, France

(Reçu le 25 juillet 1981, accepté le 4 septembre 1981)

\begin{abstract}
Résumé. - On considère le transport électronique dans des mélanges hydrogène-hélium denses, totalement ionisés, dans le cadre de la théorie de Faber-Ziman. La conductivité électrique du gaz d'électrons quasi libres et dégénérés est calculée à partir des facteurs de structure ioniques obtenus par résolution numérique des équations HNC. On envisage l'influence de la séparation de phase des mélanges $\mathrm{H}^{+}-\mathrm{He}^{2+}$ sur la conductivité.

Abstract. - Electronic transport in dense, fully ionized hydrogen-helium mixtures is investigated within the framework of the Faber-Ziman formalism. The electrical conductivity of the degenerate, nearly free electron gas is calculated, using the partial ionic structure factors obtained from numerical solutions of the HNC equations. The possible influence of the phase separation of $\mathrm{H}^{+}-\mathrm{He}^{2+}$ mixtures on the conductivity is discussed.
\end{abstract}

In fully ionized plasmas of sufficiently high density, the electron Fermi temperature $T_{\mathrm{F}}$ may greatly exceed the plasma temperature $T$; under such circumstances the transport properties of the highly degenerate, nearly free electrons can be accurately calculated in the framework of the Ziman formalism [1] $\left({ }^{1}\right)$. Such a calculation has recently been carried out for the dense hydrogen plasma [2]; for this purpose the ionic component was modelled by a classical one-component plasma (OCP), i.e. a fluid of point ions in an uniform neutralizing mechanically rigid background, while the corresponding ionic structure factors were obtained from Monte-Carlo and hypernetted chain (HNC) calculations. In this letter we extend the previous calculation to the case of $\mathrm{H}^{+}-\mathrm{He}^{2+}$ mixtures. The variation of the conductivity with temperature is calculated for fixed values of the pressure $P$, and of the $\mathrm{He}$ concentration.

The extension of the Ziman theory for electron transport to the case of alloys is straightforward [3]. It has been previously applied to the case of fully ionized $\mathrm{H}^{+}-\mathrm{He}^{2+}$ mixtures by Giordano et al. [4],

(*) Associé au C.N.R.S.

$\left({ }^{1}\right)$ which accounts for the d.c. transport coefficients through the elastic scattering of the degenerate electrons at the Fermi surface by the ion density fluctuations. on the basis of the " Mean Spherical » approximation (MSA) for the ionic structure. In that work the ions are assimilated to charged hard spheres, with the additional approximation that the effective hard sphere diameters of $\mathrm{H}^{+}$and $\mathrm{He}^{2+}$ ions are equal. The MSA procedure has the advantage of yielding analytical ionic partial structure factors, but the " hard sphere " part of the ion-ion interaction has no physical origin, and the effective hard sphere diameter must be determined independently by an ad hoc variational procedure [5]. In the present work the ionic pair structures are deduced from the very accurate hypernetted chain (HNC) theory which does not rely on the artificial introduction of an effective hard core.

In the following we denote by $n_{\mathrm{i}}$ the total ionic number density and by $C_{1}$ and $C_{2}$ the partial number concentrations of $\mathrm{H}^{+}\left(Z_{1}=1\right)$ and $\mathrm{He}^{2+}\left(Z_{2}=2\right)$ respectively. We choose the "ion sphere" radius $a_{\mathrm{i}}=\left(3 / 4 \pi n_{\mathrm{i}}\right)^{1 / 3}$ as the unit of length and define dimensionless Fourier transforms according to :

$$
\hat{f}(q)=3 \int_{0}^{\infty} \frac{\sin q x}{q x} f(x) x^{2} \mathrm{~d} x
$$

with $x=r / a_{\mathrm{i}}$ and $q=k a_{\mathrm{i}}$.

The plasma parameter is defined as usual as 
$\Gamma=\beta e^{2} / a_{\mathrm{i}}$, with $\beta=1 / k_{\mathrm{B}} T$. The degenerate electron gas is characterized by the usual dimensionless parameter $r_{\mathrm{s}}=a_{\mathrm{e}} / a_{0}$, where $a_{0}$ is the Bohr radius, $a_{\mathrm{e}}=a_{\mathrm{i}} /\langle Z\rangle^{1 / 3}$ denotes the " electron sphere " radius, with $\left\langle Z^{v}\right\rangle=C_{1} Z_{1}^{v}+C_{2} Z_{2}^{v}$. The electron gas is non relativistic, as long as $k_{\mathrm{B}} T_{\mathrm{F}}<m_{\mathrm{e}} C^{2}$, i.e. $r_{\mathrm{s}} \gtrsim 0.02$. It can be taken as essentially homogeneous as long as the Thomas-Fermi (TF) screening length exceeds the " ion-sphere » radius $a_{\mathrm{i}}$, i.e. as long as the reduced TF wave-number

$$
q_{\mathrm{TF}}=a_{\mathrm{i}} k_{\mathrm{TF}}=(12\langle Z\rangle / \pi)^{1 / 3} r_{\mathrm{s}}^{1 / 2} \lesssim 1 .
$$

Under these conditions the fully stripped " point » ions can be considered as moving in a rigid, uniform background and are well modelled by an obvious generalization of the OCP, the so-called « binary ionic mixture » (BIM) [6], where the ions interact by the unscreened Coulomb potential

$$
\beta v_{\mu v}(x)=Z_{\mu} Z_{v} \Gamma / x \quad(\mu, v=1,2) .
$$

The usual Faber-Ziman formula for the electron d.c. resistivity in binary fluid mixtures then reads :

$$
\begin{aligned}
\rho & =\frac{12 \pi}{\hbar e^{2} v_{\mathrm{F}}^{2} n_{\mathrm{i}}} \cdot \frac{1}{\left(2 q_{\mathrm{F}}\right)^{4}} \times \\
& \times \int_{0}^{2 q_{\mathrm{F}}} \mathrm{d} q q^{3} \sum_{\mu, \nu=1}^{2}\left(C_{\nu} C_{\mu}\right)^{1 / 2} \hat{U}_{\mu}(q) \hat{U}_{\nu}(q) S_{\mu \nu}(q)
\end{aligned}
$$

where $v_{\mathrm{F}}$ is the electron Fermi velocity and

$$
q_{\mathrm{F}}=a_{\mathrm{i}} k_{\mathrm{F}}=(9 \pi / 4)^{1 / 3}\langle Z\rangle^{1 / 3}
$$

the reduced Fermi wave-number. The $S_{\mu \nu}(q)$ are the partial ionic structure factors, while $\hat{U}(q)$ is the screened electron-ion Coulomb interaction :

$$
\hat{U}(q)=\frac{3 Z_{\mu} e^{2}}{a_{\mathrm{i}} \varepsilon(q) q^{2}} .
$$

The static dielectric function $\varepsilon(q)$ accounts for electron screening. In the very high density limit $\left(r_{\mathrm{s}} \ll 1\right) \varepsilon(q)$ goes over to its RPA expression :

$$
\varepsilon_{\mathrm{L}}(q)=1+\frac{q_{\mathrm{TF}}^{2}}{q^{2}} f\left(\frac{q}{2 q_{\mathrm{F}}}\right)
$$

where $f$ denotes the Lindhard function [7] :

$$
f(y)=\frac{1}{2}+\frac{\dot{1}-y^{2}}{4 y} \ln \left|\frac{1+y}{1-y}\right| \text {. }
$$

At lower density $\left(r_{\mathrm{s}} \simeq 1\right), \varepsilon_{\mathrm{L}}(q)$ must be corrected for exchange and correlation effects. We have adopted the form proposed by Geldart and Vosko [8] who replace the Lindhard function $f(y)$ by :

$$
\begin{aligned}
F(y) & =\frac{f(y)}{1+\left(\frac{q_{\mathrm{TF}}}{2 q_{\mathrm{F}}}\right)^{2} f(y)\left(2 y^{2}+g\right)^{-1}} \\
g & =\left(1+0.0262 r_{\mathrm{s}}\right)^{-1} .
\end{aligned}
$$

The second ingredient in the Faber-Ziman formula (1) is a set of three ionic partial structure factors $S_{\mu v}(q)$ which characterize the ion density fluctuations in the BIM model. They are given in terms of the Fourier transforms of the pair correlation functions :

$$
S_{\mu \nu}(q)=\delta_{\mu \nu}+\left(C_{\mu} C_{v}\right)^{1 / 2} \hat{h}_{\mu \nu}(q)
$$

where $h_{\mu v}(x)=g_{\mu v}(x)-1$, and $g_{\mu v}(x)$ denotes the standard static pair distribution functions deduced from the HNC closure equations :

$$
g_{\mu v}(x)=\exp \left\{-\Gamma Z_{\mu} Z_{v} / x+h_{\mu v}(x)-C_{\mu v}(x)\right\} .
$$

In equation (7), $C_{\mu v}(x)$ denote the direct correlation functions which are defined in terms of the $h_{\mu \nu}$ by the coupled Ornstein-Zernike relations :

$$
\hat{h}_{\mu \nu}(q)=\hat{C}_{\mu \nu}(q)+\sum_{\alpha=1}^{2} C_{\alpha} \hat{h}_{\mu \alpha}(q) \hat{C}_{\alpha \nu}(q) \text {. }
$$

Equations (7) and (8) form a closed set of three integral equations for the $g_{\mu \nu}(x)$, which are solved numerically [6]. The resulting $S_{\mu v}(q)$ agree quite well with available " exact Monte-Carlo data " [6]; they contain the temperature dependence of the resistivity in equation (1).

The calculations have been carried out at constant pressure. Let $\pi$ denotes the reduced pressure (in atomic units), $\pi=P a_{0}^{4} / e^{2}$ ( $\pi=1$ corresponds to $P=294.2$ Mbar), written as a sum

$$
\pi=\pi_{\mathrm{e}}+\pi_{\mathrm{i}}
$$

The dominant, electronic contribution is given approximately by the Nozières-Pines formula [9], complemented by a kinetic finite temperature correction :

$$
\begin{aligned}
\pi_{\mathrm{e}}=\frac{3}{8 \pi r_{\mathrm{s}}^{3}}\left[\frac{1.473}{r_{\mathrm{s}}^{2}}\right. & -\frac{0.305}{r_{\mathrm{s}}}-0.0103+ \\
& \left.+0.18 \times 10^{-10} r_{\mathrm{s}}^{2} T^{2}(\mathrm{~K})\right] .
\end{aligned}
$$

The ionic part, $\pi_{\mathrm{i}}$, is explained for the BIM model in reference [6]. For moderate charge ratios $Z_{2} / Z_{1}$, the excess internal energy of mixing of the BIM is negligible, and the ionic pressure $\pi_{i}$ is then simply expressible in terms of the equation of state of the OCP [5] :

$$
\pi_{\mathrm{i}}=\frac{3}{4 \pi} \times \frac{1}{r_{\mathrm{s}}^{4}} \times \frac{1}{\Gamma\langle Z\rangle^{2 / 3}}\left[1+\frac{C_{1}}{3} u\left(\Gamma_{1}\right)+\frac{C_{2}}{3} u\left(\Gamma_{2}\right)\right]
$$

where $u=U^{\mathrm{ex}} / N k_{\mathrm{B}} T$ denotes the reduced excess internal energy of the OCP and $\Gamma_{\mu}=\Gamma\langle Z\rangle^{1 / 3} Z_{\mu}^{5 / 3}$ [6]. Imposing a fixed total pressure $\pi$, equations (9-11) yields an implicit equation for $\Gamma$ as a function of $\pi$, $T$ and $C_{1}$, which is solved iteratively. The resulting $\Gamma$, together with $C_{1}$, determines the $S_{\mu \nu}(q)$ to be used in equation (1). 


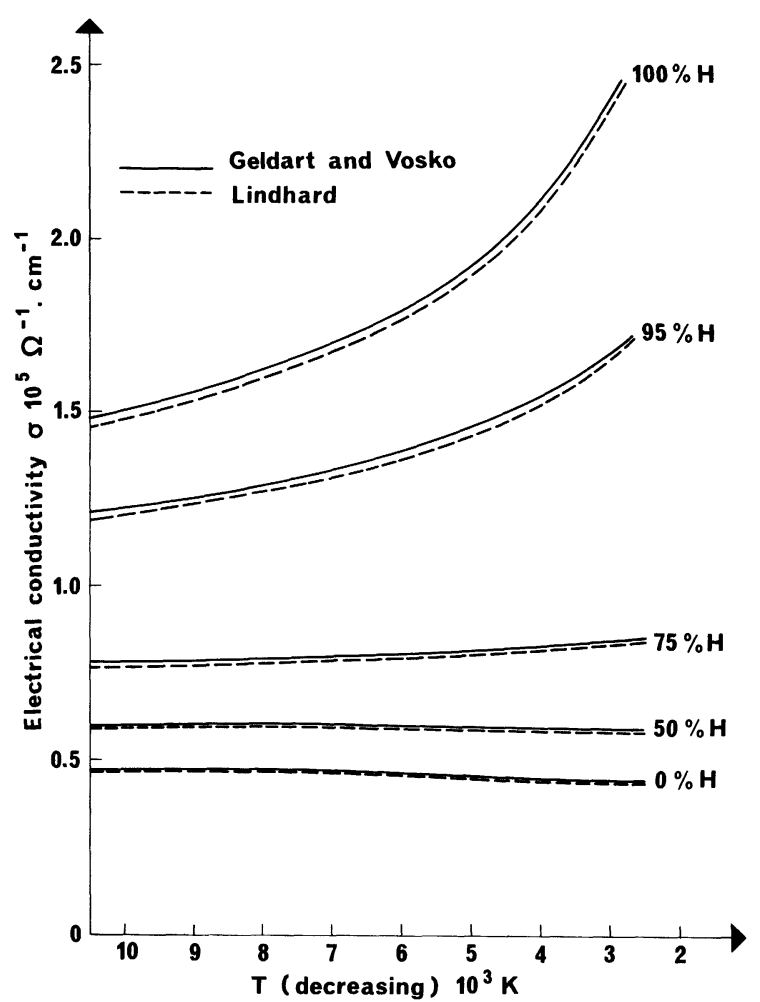

Fig. 1. - D.c. conductivity in strongly coupled H-He mixtures.

Typical results for the electrical conductivity $\sigma=1 / \rho$ are shown in figure 1 for $\pi=0.1 . C_{1}$ ranges from 0 to $100 \%$ hydrogen, while the temperature ranges from $3 \times 10^{3} \mathrm{~K}$ to $10.5 \times 10^{3} \mathrm{~K}$. Note that these physical conditions correspond roughly to those of the deep interior of Jupiter [5]. As shown by Stevenson [11] and by Hansen et al. [6], these pressure and temperature conditions lead to a phase-separation of the $\mathrm{H}^{+}-\mathrm{He}^{2+}$ mixture, and our values for $\sigma$ (at fixed $C_{1}$ and decreasing $T$ ) are crossing the demixing curve. Figure 1 displays the variation of $\sigma$ with $T$ for various concentrations $C_{1}$. The following comments are in order :

$\alpha$ ) As expected, for given $T$ and $\pi$, the resistivity of pure $\mathrm{He}^{2+}$ is considerably larger than the corresponding resistivity of pure $\mathrm{H}^{+}$; even a small amount of, say $5 \%, \mathrm{He}^{2+}$ in the mixture leads to a large drop of the d.c. conductivity, due to the much stronger scattering of electrons by the $\mathrm{He}^{2+}$ nuclei (cf. a factor $Z^{2}$, due to $\left[\hat{U}_{2}(q)\right]^{2}$, appearing in the integrand of equation (1)). Notice the important variation of $\sigma$ with $T$ in the case of pure $\mathrm{H}^{+}$, which is to be contrasted with the much smoother variation in the case of pure $\mathrm{He}^{2+}$, which has a nearly constant resistivity.

$\beta)$ There is no significant discrepancy between the curves computed on the basis of the Lindhard dielectric function, and those based on its Geldart-Vosko counterpart; note however that the differences are largest in the case of pure $\mathrm{H}^{+}$.

$\gamma)$ Upon crossing the phase-separation curve, the present calculation shows no change in the values of the electrical conductivity $\sigma$ and its temperature derivative (for $\pi=0.1$, the critical parameters are $\dot{T}_{\mathrm{c}} \simeq 6900 \mathrm{~K}$ and $C_{1} \simeq 0.71$ [6]). The values of $\sigma$ below the coexistence curve, when calculated by the Faber-Ziman formula in conjunction with the partial structure factors of the BIM model and an RPA-like dielectric function, appear as those of a metastable phase, or equivalently as the analytic continuation of the conductivity expression which is, strictly speaking, only valid above the coexistence curve, in the homogeneous phase. It should be noted, however, that the phase separation is expected to have a strong influence on the conductivity, due to the large concentration fluctuations near the coexistence curve. Such fluctuations are not properly accounted for in our calculation since the ionic and electronic density fluctuations are essentially decoupled in the BIM model. A more fundamental approach would have to consider the mixture as a three component fluid, where the ionic and electronic components are treated on an equal footing [12]. The effect of the phase separation on the electrical conductivity can however be easily illustrated within the framework of the present approach, as follows : at a sufficiently low temperature, say $T=3 \times 10^{3} \mathrm{~K}$, the $\mathrm{H}^{+}-\mathrm{He}^{2+}$ mixture separates into two phases which are practically pure $\mathrm{H}^{+}$and $\mathrm{He}^{2+}$, and which have conductivities

$$
\sigma_{1}=2.415 \times 10^{5} \Omega^{-1} \mathrm{~cm}^{-1}\left(\mathrm{H}^{+}\right)
$$

and

$$
\sigma_{2}=0.445 \times 10^{5} \Omega^{-1} \mathrm{~cm}^{-1}\left(\mathrm{He}^{2+}\right)
$$

respectively. The conductivity of the (metastable) mixture, computed as above, is

$$
\sigma=0.856 \times 10^{5} \Omega^{-1} \mathrm{~cm}^{-1}
$$

for $C_{1}=0.75$ and $T=3 \times 10^{3} \mathrm{~K}$. Now, upon applying the results of the effective medium theory [13] to a heterogeneous binary medium with randomly varying transport coefficients $\sigma_{1}$ and $\sigma_{2}$, we obtain a macroscopic conductivity $\bar{\sigma}$ given by :

$$
\begin{array}{r}
\bar{\sigma}=\frac{1}{4}\left\{\left(3 C_{2}-1\right) \sigma_{2}+\left(3 C_{1}-1\right) \sigma_{1}+\left[\left(3 C_{2}-1\right) \sigma_{2}\right.\right. \\
\left.\left.+\left(3 C_{1}-1\right) \sigma_{1}\right]^{2}+8 \sigma_{1} \sigma_{2}\right\}^{1 / 2}
\end{array}
$$

which leads to a conductivity $\bar{\sigma} \simeq 1.704 \Omega^{-1} \mathrm{~cm}^{-1}$. substantially larger than the previous one.

We are planning to extend the present analysis of electronic transport in binary ionic mixtures to higher temperatures, by incorporating the temperature dependence of the dielectric function of the partially degenerate electron gas [14]. The calculations will also be extended to transport coefficients other than the electrical conductivity, as well as to other types of higher valence « impurities » (i.e. different values of $Z_{2}$ ) than $\mathrm{He}^{2+}$.

Acknowledgments. - We thank D. Levesque, H. Minoo and J. J. Weis for useful discussions. 


\section{References}

[1] Ziman, J. M., Philos. Mag. 6 (1961) 1013.

See also : FAber, T. E., Adv. Phys. 15 (1967) 5.

[2] Minoo, H., Deutsch, C. and Hansen, J. P., Phys. Rev. A 14 (1976) 840.

See also : Deutsch, C., J. Non-Cryst. Solids 8 (1972) 713.

[3] Faber, T. E. and Ziman, J. M., Philos. Mag. 11 (1965) 153.

[4] Giordano, M., Nappi, C., De Angelis, U. and Forlani, A., Phys. Lett. 80A (1980) 342.

[5] Stevenson, D. J. and AshCroft, N. W., Phys. Rev. A 9 (1974) 782.

[6] Hansen, J. P., Torrie, G. M. and Vieillefosse, P., Phys. Rev. A 16 (1977) 2153.

Brami, B., Hansen, J. P. and Joly, F., Physica 95A (1979) 505.
[7] See e.g. March, N. H., Liquid Metals (Pergamon, New York) 1968.

[8] Geldart, D. J. and Vosko, S. H., Can. J. Phys. 44 (1966) 2137.

[9] Nozières, P. and Pines, D., Phys. Rev. 111 (1958) 442.

[10] De Witt, H. E., Phys. Rev. A 14 (1976) 1290.

[11] Stevenson, D. J., Phys. Rev. B 12 (1975) 3999.

[12] Boercker, D. B., Phys. Rev. A 23 (1981) 1969.

[13] Bruggeman, D. A., Ann. Phys. (Leipz.) 24 (1935) 636.

OdelevskiI, V. I., J. Tech. Phys. (USSR) 21 (1951) 678.

Landauer, R., J. Appl. Phys. 23 (1952) 779.

Cohen, M. H. and Jortner, J., Phys. Rev. A 10 (1974) 978.

[14] Gouedard, C. and Deutsch, C., J. Math. Phys. 18 (1978) 32. 